\title{
Comparison of cyclic fatigue resistance of different and novel heat-treated nickel-titanium rotary file systems at the intracanal temperature
}

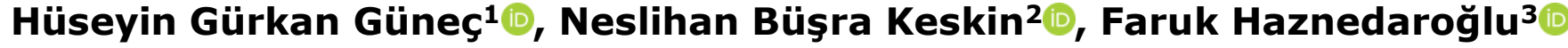

${ }^{1}$ Atlas University, Faculty of Dentistry, Department of Endodontics, Istanbul, Turkey

${ }^{2}$ Ankara Yıldırım Beyazıt University, Faculty of Dentistry, Department of Endodontics, Ankara, Turkey

${ }^{3}$ Istanbul University, Faculty of Dentistry, Department of Endodontics, Istanbul, Turkey

\section{Correspondence:}

Dr. Hüseyin Gürkan GÜNEÇ

Atlas University, Faculty of Dentistry,

Department of Endodontics, Istanbul,

Turkey.

E-mail:gunec.gurkan@gmail.com

Received: 26 January 2021

Accepted: 16 July 2021

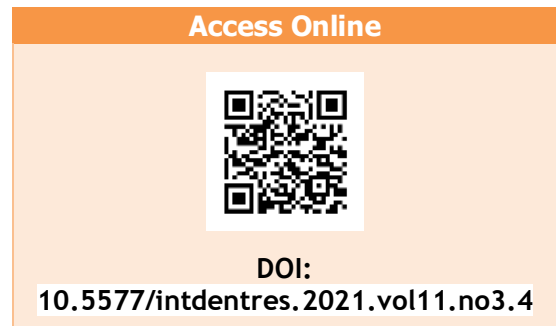

\section{Introduction}

The discovery of nickel-titanium (NiTi) rotary files in the 1980s started a new era in endodontics (1). Since $(p>0.05)$.

\section{Abstract}

Aim: The aim of this study was to compare the cyclic fatigue resistance of different heat-treated nickel-titanium rotary systems at intracanal temperature.

Methodology: A total of 90 OneCurve (Micro-Mega, Besançon, France), VDW.ROTATE (VDW Dental, Munich, Germany), Typhoon (Clinician's Choice, New Milford, CT, USA), HyFlex EDM (Coltene/Whaledent AG, Altstatten, Switzerland), and EndoArt Gold and Blue (Inci Dental, Istanbul, Turkey) $(n=15)$ rotary files $(\# 25 / 0.06)$ were tested at intracanal temperature $\left(35.5^{\circ} \mathrm{C}\right)$ using a dynamic model in a stainless-steel artificial canal with an inner diameter of $1.5 \mathrm{~mm}, 60^{\circ}$ angle of curvature, and $2 \mathrm{~mm}$ radius of curvature. Testing was conducted until fracturing, at which time the device stopped automatically, and the number of rotations was calculated as seconds. Lengths of fractured parts were measured using a digital caliper. One-way ANOVA test followed by Tukey's test was used to compare the groups. Scanning electron microscopic evaluation was performed to confirm the types of fracture.

Results: EndoArt Blue group had a significantly higher mean time to fracture in all groups, followed by the HyFlex EDM, VDW.ROTATE, OneCurve, EndoArt Gold, and Typhoon. In addition, the HyFlex EDM and VDW.ROTATE groups had no significant differences between each other and were significantly better than the others. No significant differences were found between the OneCurve, EndoArt Gold, and Typhoon groups

Conclusion: This is the first study in the literature for EndoArt NiTi files and the second study for VDW.ROTATE that evaluated cyclic fatigue resistance. Novel EndoArt Blue files exhibited significantly greater cyclic fatigue resistance than the other NiTi files.

Keywords: NiTi file, heat treatment (gold/blue), cyclic fatigue, scanning electron microscopy, intracanal temperature

How to cite this article: Güneç HG, Keskin NB, Haznedaroğlu F. Comparison of cyclic fatigue resistance of different and novel heat-treated nickel-titanium rotary file systems at intracanal temperature. Int Dent Res 2021;11(3):158-64.

https://doi.org/10.5577/intdentres.2021.vol11.no3.4 then, the use of $\mathrm{NiTi}$ rotary files for root canal preparations is becoming more prominent each day because $\mathrm{NiTi}$ files are not stronger but are more flexible than stainless-steel instruments $(2,3)$. The use of 
stainless steel may lead to several problems, including ledges, perforations, zips, and straightened root canals. These complications appear less frequently with the use of NiTi rotary files $(3,4)$. However, several reports have suggested that $\mathrm{NiTi}$ endodontic files are significantly more prone to fractures, especially during the shaping of curved or narrow root canals, which might impact the success of the root canal treatment. $\mathrm{NiTi}$ files are reported to be up to seven times more likely to fracture compared to stainless-steel files (5, 6). Many underlying factors contribute to these fractures; however, the most common reasons for $\mathrm{NiTi}$ fractures are either torsional or due to cyclic fatigue. Cyclic fatigue leads to the deterioration of the file with continuous cycles of tension, and compression happens while bending, which results in structural breakdown and fractures in the instrument. In the clinical situation, this usually occurs while shaping severely curved canals. In narrow but straight canal instrument fractures due to torsional stress, and in curved and narrow canals instrument fractures due to both torsional and flexural stress $(3,4,7)$.

Experiencing these frequent fractures causes major setbacks for root canal treatments in many clinics. Therefore, several experimental studies have been conducted to prevent the fracturing of NiTi rotary files and to improve their mechanical properties $(6,8)$. These studies have included altering the operating speed, changing the file design, and attempting various metal treatments $(9,10)$. There are two crystal states of the NiTi alloy that are temperature-dependent: martensite and austenite, and the characteristics of the alloy are different in each state. Austenite is a more rigid structure and has shape memory properties, while with martensite, the NiTi alloy appears to be more flexible. When the temperature is raised above the transformation temperature, $\mathrm{NiTi}$ is converted from the martensite form into the austenite form $(8,11)$. The newly developed NiTi alloys have a transitional temperature close to body temperature, which is higher than conventional NiTi alloys. Due to the shape memory properties of the austenite state, NiTi files that are manufactured using heat treatments have been shown to exhibit much stronger cyclic fatigue resistance than conventional $\mathrm{NiTi}$ files $(8,9,12)$.

Several new-generation NiTi files were designed and marketed for endodontic treatments using a singlefile technique in perpetual rotations6. The HyFlex EDM is one of the cutting-edge instruments produced for this purpose, which is manufactured using the technique of electrical discharge machining with a controlledmemory feature $(1,13,14)$. Another instrument developed in 2017 is OneCurve which is also a singlefile NiTi system manufactured from $\mathrm{C}$-wire through the use of heat treatment (10). The Typhoon Infinite Flex instrument (Clinician's Choice Dental Products, New Milford, CT, USA), also manufactured with $\mathrm{NiTi}$ controlled-memory (CM) wire, was more fatigueresistant than instruments manufactured with $\mathrm{NiTi} M$ Wire and stronger than conventional alloy instruments $(15,16)$. The VDW.ROTATE was produced by VDW company (VDW Dental, Munich, Germany) in 2019 that manufactured a new heat treatment. The company explains in detail that to increase flexibility without compromising cutting efficiency, they adapted an S cross-section for better cutting efficiency and increased flexibility that respects more the canal anatomy (17).

The last instrument in our study was the novel and new EndoArt Blue and EndoArt Gold files developed by İnci Dental (Istanbul, Turkey) in 2019. The EndoArt NiTi file is made into a safety instrument with heat treatment, which gives them the names of Blue and Gold. However, both of them have triangular crosssections, fixed tapers, different tip sizes, and CM wires. The EndoArt Blue files are produced at a temperature of about $500-600{ }^{\circ} \mathrm{C}$, which is higher than EndoArt Gold files, which are produced at $300-350{ }^{\circ} \mathrm{C}$. They have been shown to allow for excellent preparation in the canal due to the increased flexibility, and the new $\mathrm{NiTi}$ wires have shown significantly better cyclic fatigue resistance.

This study aimed to compare the cyclic fatigue resistance of six different rotary $\mathrm{NiTi}$ files with different metallurgic and heat treatment properties (OneCurve, VDW.ROTATE, Typhoon, EndoArt Gold, EndoArt Blue, and HyFlex EDM) during instrumentation and irrigation with $\mathrm{NaOCl}$ solution at intracanal temperature $\left(35.5^{\circ} \mathrm{C}\right)$. The null hypothesis of this study is that rotary files with different metallurgic properties will have the same levels of cyclic fatigue resistance.

\section{Materials and Methods}

For this study, $90 \mathrm{NiTi}(\mathrm{n}=15)$ rotary files (\#25/0.06) were included that planned to be used in the root canal preparation, dividing them into groups: Group 1, EndoArt Blue; Group 2, OneCurve; Group 3, HyFlex EDM; Group 4, VDW.ROTATE; Group 5, Typhoon; Group 6, EndoArt Gold. The sample calculation was assessed using G*Power v3.1 for Mac (Heinrich Heine, University of Dusseldorf). Power calculation was performed for using an $F$ test: Fixed effects, one-way analysis with $\alpha=0.05$ and $B=0.95$. The results showed that there were 0.48 effect size and 0.95 power value for six groups and 90 total samples. The presence of a defect or not on the surface of the rotary files was examined under 20x magnification using a stereomicroscope (Olympus BX43; Olympus Co., Tokyo, Japan). The rotary files without any defects were used in the cyclic fatigue resistance test.

\section{Testing Device}

The dynamic cyclic fatigue resistance test was performed as a model at intracanal temperature $\left(35^{\circ} \mathrm{C}\right.$ \pm 1 ) (Fig. 1). In our experimental setup, a stone (wire) resistor and thermostat were used to increase the temperature of the artificial canal or metal block to $35.5{ }^{\circ} \mathrm{C}$ and to maintain this temperature. The temperature was monitored throughout the experiment using a digital thermometer. Current was passed through a 27-ohm $5 \mathrm{~W}$ stone resistor by a voltage source, and the stone resistor transferred the waste heat it generated to the metal block in contact with 
the transmission, causing the temperature of the metal block to increase. After the temperature reached 35.5 ${ }^{\circ} \mathrm{C}$, a thermostat was used to stop further increases and maintain the temperature. Thermostats work by breaking the circuit (creating an open circuit) when a certain temperature is reached, thus preventing current from passing through the resistor. When the metal block's temperature drops below the desired value, the circuit becomes operational again. The files were fixed on the micromotor and the contra-angle handpiece with torque and velocity set at a $6: 1$ reduction (VDW Dental, Munich, Germany). They were then connected to the testing device and used at the velocity and torque values recommended by the manufacturers.

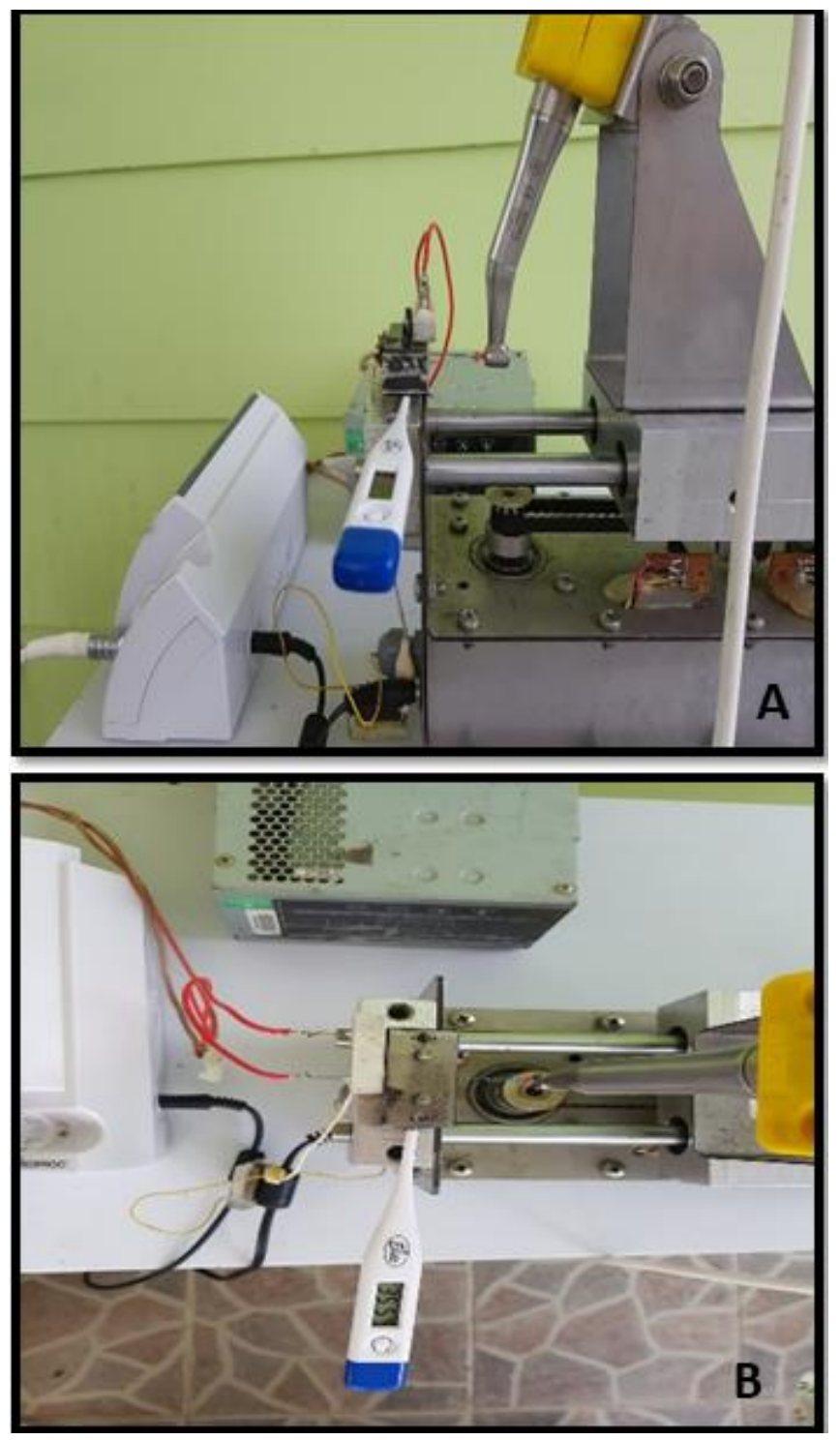

Figure 1. The dynamic cyclic fatigue testing device (A-B)

To mimic clinical conditions, $2 \mathrm{~mL}$ of $5.25 \%$ sodium hypochlorite ( $\mathrm{NaOCl}$; CanalPro; Coltene-Whaledent AG, Allstetten, Switzerland) was injected into the artificial canals, and after synthetic oil (WD-40 Company, Milton Keynes, England) was used for lubrication. All files were rotated until a fracture occurred at a $60^{\circ}$
Curvature angle inside the artificial canal with a $2 \mathrm{~mm}$ radius (Fig. 2). The instruments fixed was pushed and pulled from the canal at a $3 \mathrm{~mm} / \mathrm{s}$ velocity to mimic clinical use. At the moment of fracture, the device stopped automatically, and the number of rotations was calculated as seconds. The number of cycles to fracture (NCF) was calculated by multiplying time to fracture and rotational speed (NCF = Time to fracture $(\mathrm{min}) \times$ rotation per minute $(\mathrm{rpm}))$. The lengths of the fractured $(F L)$ parts were measured using a digital caliper.

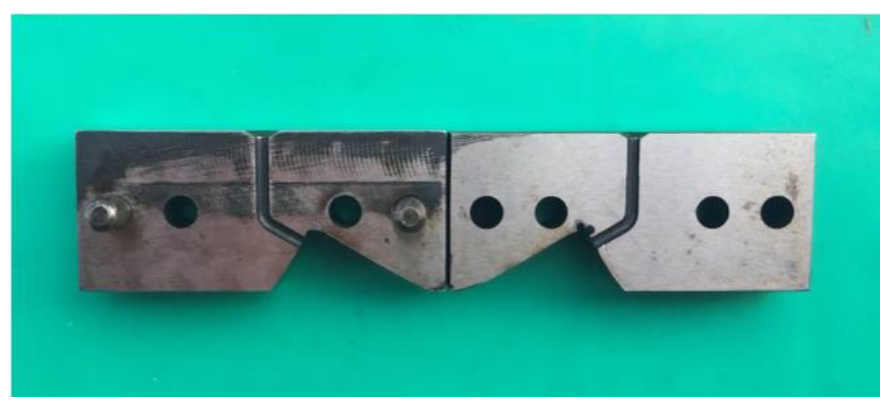

Figure 2. Artificial canal (metal block)

\section{Scanning Electron Microscopy (SEM)}

To perform this analysis, 12 pieces of fractured files (two pieces from each group) were randomly selected and examined under a scanning electron microscope (SEM; Quanta $450 \mathrm{FEI}$, Hillsboro, Oregon, USA) to confirm the type of fracture as flexural. The photomicrographs were taken from the fractured surfaces under different magnifications (150x to $3000 \mathrm{x})$ to show the presence of fracture starting points and fatigue lines where the breakage occurred, confirming cyclic fatigue (Fig. 3). A single operator performed all experimental procedures to eliminate operator bias.
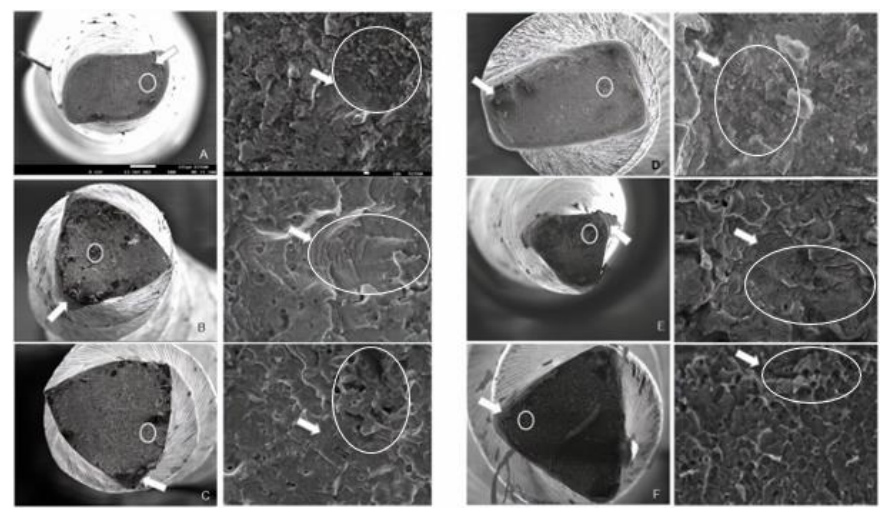

Figure 3. Scanning electron micrographs of the fracture surface after cyclic fatigue test: (A) VDW.ROTATE; (B) EndoArt Blue; (C) EndoArt Gold; (D) HyFlex EDM; (E) OneCurve; and $(F)$ Typhoon (Left: 150x magnification; Right: 3000x magnification) 


\section{Statistical analysis}

The data were evaluated using IBM SPSS version 21 software (IBM SPSS Inc., Armonk, NY, USA). Following confirmation of normal distribution of the data using the Shapiro-Wilk test. One-way ANOVA test followed by Tukey's test was used to compare the groups. Significance was established at the $95 \%$ confidence level.

\section{Results}

The mean and standard deviation of the number of cycles to failure (NCF) and fracture length (FL) of the groups tested were shown in Table 1.
In the $2 \mathrm{~mm}$ radius of the canal curvature, the novel EndoArt Blue group had a significantly higher mean time to fracture in all groups, followed by the HyFlex EDM and VDW.ROTATE groups (Table 1).

No significant differences were found between the HyFlex EDM and VDW.ROTATE groups ( $p>.05$ ). The OneCurve, EndoArt Gold, and Typhoon groups were significantly lower than the HyFlex EDM and VDW.ROTATE groups $(p<.05)$. No significant differences were found between the OneCurve, EndoArt Gold, and Typhoon groups $(p>.05)$. There were no statistically significant differences among the groups in the fracture lengths $(p>.05)$.

Table 1. The means and standard deviations of the number of cycles to failure (NCF) and fracture length (FL) of instruments in $5.25 \% \mathrm{NaOCl}$. (Mean \pm SD)

\begin{tabular}{|c|cc|}
\hline Instrument & NCF & FL \\
\hline EndoArt Blue & $25103.11 \pm 1280.452 x$ & $3.36 \pm 0.2 x$ \\
\hline Hyflex EDM & $17315.11 \pm 3597.726 y$ & $3.36 \pm 0.2 x$ \\
\hline ROTATE & $14205.33 \pm 528.254 y$ & $3.35 \pm 0.1 x$ \\
\hline One Curve & $9070.67 \pm 528.19 z$ & $3.32 \pm 0.3 x$ \\
\hline EndoArt Gold & $7711.56 \pm 552.54 z$ & $3.35 \pm 0.3 x$ \\
\hline Typhoon & $7686.20 \pm 570.097 z$ & $3.33 \pm 0.2 x$ \\
\hline
\end{tabular}

Within same column, different letters indicate groups statistically different from each other based on post hoc comparisons by Tukey test $(p<.05)$. ( $x . y . z$ columns; $a, b, c$, rows)

\section{Discussion}

Standardized parameters were used in this study, including $35.5^{\circ} \mathrm{C}$ intracanal temperature, $60^{\circ}$ angle of curvature, $2 \mathrm{~mm}$ root canal diameter, and $3 \mathrm{~mm} / \mathrm{s}$ rotation speed. These variables are also crucial in determining the lifespan and cyclic fatigue resistance of NiTi files. In experiments conducted under different conditions, a different NiTi file might exhibit a longer duration without fractures $(11,18)$.

Different methods have been used to evaluate the cyclic fatigue resistance of NiTi files $(12,19)$. A cyclic fatigue test can be performed to fracture resistance using either a static or dynamic model (20), but typically, a static model is used under well-controlled experimental conditions. Studies have revealed that the number of cycles to fracture significantly increased when using dynamic models, regardless of the brand or manufacturing process $(14,21,22)$. This is perhaps because with a static testing model, the instrument does not move back and forth, so stresses accumulate at a single point, whereas in the dynamic model, stresses are distributed along the cutting part of the instrument, which increases the resistance to cyclic fatigue (22-24). We chose a dynamic testing model to evaluate cyclic fatigue resistance of the $\mathrm{NiTi}$ instruments. It has also been reported that in the dynamic testing model, the back-and-forth motion should be limited to 1,2 , or $3 \mathrm{~mm} / \mathrm{s}$ to simulate clinical conditions $(16,25,26)$. In our study, $3 \mathrm{~mm} / \mathrm{s}$ motion was selected in respect to previous studies (27-29).

Under our testing conditions, the corrosive effects of the $\mathrm{NaOCl}$ solution did not significantly reduce the cyclic fatigue resistance of the NiTi files examined, as had been previously reported $(30,31)$. Another study reported that dynamic immersion in $\mathrm{NaOCl}$ for $1,3,5$, or 10 minutes did not significantly decrease the cyclic fatigue resistance of $\mathrm{NiTi}(18)$. Contrary to these findings, a few studies have achieved different results with dynamic immersion (32). In many previous studies, synthetic oil was used instead of $\mathrm{NaOCl}$ due to the corrosive effects of $\mathrm{NaOCl}(3,4,33)$. However, in common clinical routines, we must clean the canals with $\mathrm{NaOCl}$ to remove biofilms, so we need to use lubricants to reduce the fracture resistance of $\mathrm{NiTi}$ instruments in root canal treatments (14). To simulate these conditions, we used synthetic oil as a lubricant and used $\mathrm{NaOCl}$ as an irrigant in our test model. 
To examine the deformations that did occur, we decided to use SEM because it was the preferred imaging method used in previous studies (19). The files were evaluated in terms of metal fatigue in the direct test setup without any use in the clinic. Thus, other deformations that may occur due to the use of files were eliminated. Any signs of deformation in the SEM images taken of the broken files were seen on the fracture surfaces, and it was determined that the file surface was suddenly broken without any previous deformation (34).

It is important to note that other factors, in addition to metallurgic properties, might influence the cyclic fatigue resistance of an instrument $(7,35)$. Factors including the size and taper (21), type of metal alloy (22), heat treatments (24), cross-sectional shape (36), design (25), and kinematics (26) of the instrument influence its flexibility and cyclic lifespan. Strikingly, in our study, the EndoArt Blue displayed an approximate $50 \%$ increase in lifespan compared to the second-best instruments, the HyFlex EDM and VDW.ROTATE.

Only one previous study was found in the literature that examined the VDW.ROTATE instruments, and it had similar results to ours. The other study evaluated the cyclic fatigue resistance of the VDW.ROTATE instrument in comparison to the same size OneCurve, TF adaptive, and Mtwo instruments, and the VDW.ROTATE tendered higher cyclic fatigue resistance than the others (17).

The HyFlex EDM file is produced by electrodischarge machining, which leads to a remarkable melting appearance with microvugs instead of machining grooves and irregularities on the surface of the HyFlex EDM. This may be the reason why the HyFlex EDM presented significantly better cyclic fatigue resistance than the HyFlex CM and low deformation was seen after multiple canal instrumentations $(13,37)$. The cyclic fatigue test showed an increase in fatigue resistance of up to $700 \%$ on the HyFlex EDM compared to HyFlex CM files, is in agreement with many studies $(4,13)$. HyFlex EDM files also had greater cyclic fatigue resistance values than OneCurve files (28). In one previous study, HyFlex CM was shown to have better cyclic fatigue resistance than the Typhoon $C M$, which also corroborates our study (6).

The material's characteristics also have a substantial impact on fatigue lifetime. NiTi files made from CM wire had a significantly higher NCF than conventional NiTi wire files with similar designs (7). With the same amount of precycling, the cyclic fatigue life of the Typhoon CM files was significantly higher than that of the Typhoon files. In addition, the torque value of Typhoon CM corresponded to Typhoon files, so the larger instruments were not only less resistant to cyclic fatigue but were also most impacted by pretensioning. The type of $\mathrm{NiTi}$ metal alloy influenced cyclic fatigue resistance under various environments in other studies as well (15). Typhoon CM files generally have a significantly higher NCF in flexural fatigue, so they can normally be used in curved and narrow canals (29). In a 2014 study, the mean NCF values were $150 \%$ higher for Typhoon files and 390\% higher for the HyFlex files compared with other instruments, indicating that $\mathrm{CM}$ technologies increased the fatigue resistance of rotary NiTi files. Despite the Typhoon's good values, our findings were similar to Braga et al.'s (38).

OneCurve is the first new-generation NiTi file and was designed in 2017 with a single-file technique (10). It is clear from our results that OneCurve had one of the lowest resistances to cyclic fatigue among the files tested, suggesting that the technology has already advanced significantly in just those few years. Similarly, Typhoon-which represents one of the latest generations of $\mathrm{CM} \mathrm{NiTi}$ files-also exhibited a much shorter lifespan compared to newer products such as EndoArt Blue. Interestingly, while EndoArt Blue is the instrument with the highest cyclic fatigue resistance, a novel EndoArt Gold is grouped with two of the weakest $\mathrm{NiTi}$ files tested in our study. However, this is expected according to the manufacturer's statements and is also consistent with a previous report suggesting that blue treatment significantly improved cyclic fatigue resistance in new-generation NiTi rotary files (39).

Despite the limitations of this study, EndoArt Blue has stronger cyclic fatigue resistance compared to five other new-generation $\mathrm{NiTi}$ rotary files (OneCurve, VDW.ROTATE, Typhoon, EndoArt Gold, HyFlex EDM). This finding is novel because the cyclic fatigue resistance of EndoArt Blue has never been compared with other NiTi files. The most likely explanation for this difference is the potentially varying $\mathrm{C}$-wire based heat treatment techniques during the manufacture of these blue NiTi files, which is in agreement with previous studies $(10,31,40)$. According to the manufacturer, the EndoArt Blue is made with heat treatment at more than $500{ }^{\circ} \mathrm{C}$ in order to have more flexible $C M$ wire and better cyclic fatigue values. Therefore, the transformation temperature of $\mathrm{NiTi}$ alloy used for producing EndoArt Blue may be higher compared to NiTi alloy for other rotary files, and the differences in heat treatment techniques might make EndoArt Blue more resistant to cyclic fatigue, specifically at intracanal temperature. However, further research is needed to verify whether EndoArt Blue has a longer lifespan in other conditions or compared to other new-generation NiTi files that are not included in this study.

\section{Conclusions}

Within the limitations of our study, we found that the novel EndoArt Blue files displayed significantly better cyclic fatigue resistance and hence superior fracture resistance when compared to HyFlex EDM, VDW.ROTATE, OneCurve, EndoArt Gold, and Typhoon files when tested with a $2 \mathrm{~mm}$ radius of curvature and a 600 angle in an artificial canal at intracanal temperature. Future studies should focus on conducting different tests and comparing numerous new heat-treated rotary files. 
Peer-review: Externally peer-reviewed.

Author Contributions: Conception - H.G.G.; Design - N.B.K., F.H.; Supervision - H.G.G.; Materials - N.B.K., F.H.; Data Collection and/or Processing - H.G.G., N.B.K.; Analysis and/or Interpretation - F.H.; Literature Review - H.G.G., F.H.; Writer - N.B.K.; F.H.; Critical Review -H.G.G.

Conflict of Interest: No conflict of interest was declared by the authors.

Financial Disclosure: The authors declared that this study has received no financial support.

\section{References}

1. Yilmaz K, Uslu G, Ozyurek T. In vitro comparison of the cyclic fatigue resistance of HyFlex EDM, One G, and ProGlider nickel titanium glide path instruments in single and double curvature canals. Restor Dent Endod 2017;42:282-9. (Crossref)

2. Gavini G, Santos MD, Caldeira CL, Machado MEL, Freire LG, Iglecias EF, et al. Nickel-titanium instruments in endodontics: a concise review of the state of the art. Braz Oral Res 2018;32:e67. (Crossref)

3. Gundogar M, Ozyurek T. Cyclic fatigue resistance of OneShape, HyFlex EDM, WaveOne Gold, and Reciproc Blue nickel-titanium instruments. J Endod 2017;43:1192-96. (Crossref)

4. Pedulla E, Lo Savio F, Boninelli S, Plotino G, Grande NM, La Rosa G, et al. Torsional and cyclic fatigue resistance of a new Nickel-Titanium instrument manufactured by electrical discharge machining. J Endod 2016;42:156-9. (Crossref)

5. Gambarini G, Plotino G, Grande NM, AlSudani D, De Luca M, Testarelli L. Mechanical properties of nickel-titanium rotary instruments produced with a new manufacturing technique. Int Endod J 2011;44:337-41. (Crossref)

6. Modesto TC, Acosta ECP, Resende PD, Pereira ESJ, Peixoto I, Buono VTL, et al. Cyclic flexural fatigue resistance of $\mathrm{NiTi}$ controlled memory and blue technology instruments after torsional preloading. J App Oral Sci 2018;26:e20180144. (Crossref)

7. Shen Y, Qian W, Abtin H, Gao Y, Haapasalo M. Fatigue testing of controlled memory wire nickel-titanium rotary instruments. J Endod 2001;37:997-1001. (Crossref)

8. Otsuka K, Wayman CM. Shape memory materials. 1st ed. Cambridge (UK): Cambridge University Press; 1998.

9. Staffoli S, Grande NM, Plotino G, Ozyurek T, Gundogar M, Fortunato $L$, et al. Influence of environmental temperature, heat-treatment and design on the cyclic fatigue resistance of three generations of a single-file nickel-titanium rotary instrument. Odontology 2019;107:301-7. (Crossref)

10. Elnaghy AM, Elsaka SE. Cyclic fatigue resistance of One Curve, 2Shape, ProFile Vortex, Vortex Blue, and RaCe nickel-titanium rotary instruments in single and double curvature canals. J Endod 2018;44:1725-30. (Crossref)

11. Gambarini G, Piasecki L, Miccoli G, Gaimari G, Di Giorgio R, Di Nardo D, et al. Classification and cyclic fatigue evaluation of new kinematics for endodontic instruments. Aust Endod J 2019;45:154-62. (Crossref)

12. Larsen CM, Watanabe I, Glickman GN, He J. Cyclic fatigue analysis of a new generation of nickel titanium rotary instruments. J Endod 2009;35:401-3. (Crossref)

13. Pirani C, lacono F, Generali L, Sassatelli P, Nucci C, Lusvarghi $L$, et al. HyFlex EDM: superficial features, metallurgical analysis and fatigue resistance of innovative electro discharge machined NiTi rotary instruments. Int Endod J 2016;49:48393. (Crossref)

14. Bhatt A, Rajkumar B. A Comparative evaluation of cyclic fatigue resistance for different endodontic NiTi rotary files:
An in-vitro study. J Oral Biol Craniofac Res 2019;9:119-21. (Crossref)

15. Shen Y, Qian W, Abtin H, Gao Y, Haapasalo M. Effect of environment on fatigue failure of controlled memory wire nickel-titanium rotary instruments. J Endod 2012;38:376-80. (Crossref)

16. Zubizarreta-Macho Á, Mena Álvarez J, Albaladejo Martínez A, Segura-Egea JJ, Caviedes Brucheli J, Agustín-Panadero R, et al. Influence of the pecking motion frequency on the cyclic fatigue resistance of endodontic rotary files. J Clin Med 2019;9:45. (Crossref)

17. Ertugrul IF, Orhan EO. Cyclic fatigue and energy-dispersive Xray spectroscopy examination of the novel ROTATE instrument. Microsc Res Tec 2019;82:2042-48. (Crossref)

18. Pedullà É, Benites A, La Rosa GM, Plotino G, Grande NM, Rapisarda E, et al. Cyclic fatigue resistance of Heat-Treated Nickel-Titanium instruments after immersion in sodium hypochlorite and/or sterilization. J Endod 2018;44:648-53. (Crossref)

19. Al Shwaimi E. Cyclic fatigue resistance of a novel rotary file manufactured using controlled memory $\mathrm{Ni}-\mathrm{Ti}$ technology compared to a file made from $\mathrm{M}$-wire file. Int Endod $\mathrm{J}$ 2018;51:112-7. (Crossref)

20. Keleș A, Eymirli A, Uyanık O, Nagas E. Influence of Static and Dynamic cyclic fatigue tests on the lifespan of four reciprocating systems at different temperatures. Int Endod J 2019;52:880-6. (Crossref)

21. Tanomaru-Filho M, Espir CG, Venção AC, Macedo-Serrano N, Camilo-Pinto J, Guerreiro-Tanomaru JM. Cyclic fatigue resistance of Heat-Treated Nickel-Titanium instruments. Iran Endod J 2018;13:312-7.

22. El Feky HM, Ezzat KM, Bedier MMA. Cyclic fatigue resistance of $\mathrm{M}$-Pro and $\mathrm{RaCe} \mathrm{Ni}$-Ti rotary endodontic instruments in artificial curved canals: a comparative in vitro study. Restor Dent Endod 2019;44:44. (Crossref)

23. Rubio J, Zarzosa JI, Pallarés A. A Comparative study of cyclic fatigue of 10 different types of endodontic instruments: An in vitro study. Acta Stomatol Croat 2019;53:28-36. (Crossref)

24. Gambarini G, Galli M, Di Nardo D, Seracchiani M, Donfrancesco $\mathrm{O}$, Testarelli L. Differences in cyclic fatigue lifespan between two different heat treated $\mathrm{NiTi}$ endodontic rotary instruments: WaveOne Gold vs EdgeOne Fire. J Clin Exp Dent 2019;11:609-13. (Crossref)

25. Plotino G, Grande NM, Testarelli L, Gambarini G. Cyclic fatigue of Reciproc and WaveOne reciprocating instruments. Int Endod J 2012;45:614-8. (Crossref)

26. Gambarini G, Piasecki L, Miccoli G, Gaimari G, Di Giorgio R, Di Nardo $D$, et al. Classification and cyclic fatigue evaluation of new kinematics for endodontic instruments. Aust Endod J 2019;45:154-62. (Crossref)

27. Kwak SW, Lee JY, Goo HJ, Kim HC. Effect of surface treatment on the mechanical properties of Nickel-Titanium files with a similar cross-section. Restor Dent Endod 2017;42:216-23. (Crossref)

28. Uygun AD, Unal M, Falakaloglu S, Guven Y. Comparison of the cyclic fatigue resistance of hyflex EDM, vortex blue, protaper gold, and onecurve nickel-titanium instruments. Niger J Clin Pract 2020;23:41-5.

29. Acosta EC, Resende PD, Peixoto IF, Pereira ÉS, Buono VT, Bahia MG. Influence of cyclic flexural deformation on the torsional resistance of controlled memory and conventional nickeltitanium instruments. J Endod 2017;43:613-8. (Crossref)

30. Cai JJ, Tang N, Ge JY. Effect of irrigation on surface roughness and fatigue resistance of controlled memory wire NickelTitanium instruments. Int Endod J 2017;50:718-24. (Crossref)

31. Arias A, Hejlawy S, Murphy S, de la Macorra JC, Govindjee S, Peters OA. Variable impact by ambient temperature on fatigue resistance of Heat-Treated nickel titanium instruments. Clin Oral Invest 2019;23:1101-8. (Crossref) 
32. Palma PJ, Messias A, Cerqueira AR, Tavares LD, Caramelo F, Roseiro $L$, et al. Cyclic fatigue resistance of three rotary file systems in a Dynamic model after immersion in sodium hypochlorite. Odontology 2019;107:324-32. (Crossref)

33. Topçuoğlu HS, Topçuoğlu G, Akti A, Düzgün S. In vitro comparison of cyclic fatigue resistance of ProTaper Next, HyFlex CM, OneShape, and ProTaper Universal instruments in a canal with a double curvature. J Endod 2016;42:969-71. (Crossref)

34. Uslu G, Özyürek T, Yılmaz K, Gündoğar M. Cyclic fatigue resistance of R-Pilot, HyFlex EDM and PathFile nickel-titanium glide path files in artificial canals with double (S-shaped) curvature. Int Endod J 2018;51:584-9. (Crossref)

35. Vieira EP, Pereira ES, Peixoto IF, Buono VT, Bahia MG. Fatigue resistance of rotary ProTaper Universal instruments after use with and without lateral pressure motion. Arq Odontol 2015;51:7-13.

36. Gambarini G, Miccoli G, Seracchiani M, Khrenova T, Donfrancesco O, D'Angelo, et al. Role of the flat-designed surface in improving the cyclic fatigue resistance of endodontic $\mathrm{NiTi}$ rotary instruments. Materials (Basel) 2019;12:23-5. (Crossref)

37. Wang FF, Yang YJ, Hou XM. Surface microstructure and cyclic fatigue resistance of electro discharged machining nickeltitanium endodontic instrument. Beijing Da Xue Xue Bao Yi Xue Ban 2018;50:876-81.

38. Braga LC, Faria Silva AC, Buono VT, de Azevedo Bahia MG. Impact of heat treatments on the fatigue resistance of different rotary nickel-titanium instruments. J Endod 2014;40:1494-7. (Crossref)

39. Plotino G, Grande NM, Cotti E, Testarelli L, Gambarini G. Blue treatment enhances cyclic fatigue resistance of vortex nickeltitanium rotary files. J Endod 2014;40:1451-71. (Crossref)

40. Plotino G, Grande NM, Testarelli L, Gambarini G, Castagnola R, Rossetti A, et al. Cyclic Fatigue of Reciproc and Reciproc Blue Nickel-titanium Reciprocating Files at Different Environmental Temperatures. J Endod 2018;44:1549-52. (Crossref) 